\title{
Internal Model Control for a Light-Weight Active Noise-Reducing Casing
}

\author{
Krzysztof MAZUR, Marek PAWEŁCZYK \\ Institute of Automatic Control \\ Silesian University of Technology \\ Akademicka 16, 44-100 Gliwice, Poland; e-mail: \{Krzysztof.Jan.Mazur, Marek.Pawelczyk\}@polsl.pl
}

(received March 10, 2016; accepted April 15, 2016)

\begin{abstract}
The active noise-reducing casing developed and promoted by the authors in recent publications have multiple advantages over other active noise control methods. When compared to classical solutions, it allows for obtaining global reduction of noise generated by a device enclosed in the casing. Moreover, the system does not require loudspeakers, and much smaller actuators attached to the casing walls are used instead. In turn, when compared to passive casings, the walls can be made thinner, lighter and with much better thermal transfer than sound-absorbing materials. For active noise control a feedforward structure is usually used. However, it requires an in-advance reference signal, which can be difficult to be acquired for some applications. Fortunately, usually the dominant noise components are due to rotational operations of the enclosed device parts, and thus they are tonal and multitonal. Therefore, it can be adequately predicted and the Internal Model Control structure can be used to benefit from algorithms well developed for feedforward systems. The authors have already tested that approach for a rigid casing, where interaction of the walls was significantly reduced. In this paper the idea is further explored and applied for a light-weight casing, more frequently met in practice, where each vibrating wall of the casing influences all the other walls. The system is verified in laboratory experiments.
\end{abstract}

Keywords: active noise-vibration control, active structural acoustic control, active casing, Internal Model Control.

\section{Introduction}

The active noise-reducing casing, generally proposed in (FULLER et al., 1994) and developed and promoted by the authors (MAZUR, PAWELCZYK, 2015a; Wrona, PAWELCZYK, 2014) have multiple advantages over other active and passive noise control methods. Compared to classical Active Noise Control systems it allows for obtaining global noise reduction. Compared to passive barriers, it can be very effective for reduction of low-frequency noise, which is a major problem both in working and living environments (SHEHAP et al., 2016), even with thin walls. Many devices already use casings made of thin aluminum or steel plates and such casing can be retrofitted with actuators for active control. A various types of actuators can be used, for example MFC patches become very popular due to high power-to-mass ratio (LENIOWSKA, MAZAN, 2015; GóRski, Kozupa, 2012; Mazur, PAwelczyK, 2011) and the influence of such elements on the casing is minimal. However, the most effective d33 effect MFC patches are nonlinear and more complex control algorithms are needed for high performance (MAZuR, PAWELCZYK, 2013a; 2013b). Additionally, the d33 effect MFC patches require very high voltages, up to $1500 \mathrm{~V}$. When the actuator mass is not important, electro-dynamic actuators can be used. However, for thin plates their mass must be taken into account (Wrona et al., 2014). Retrofitting the casing with actuators does not reduce the heat transfer from the device, which can be a problem when a thick passive casing is used. With thermal isolation caused by passive acoustic isolation, the device may start to overheat and an additional cooling system, which may also generate noise, is needed.

The sound radiation by various structures and its reduction have been widely studied in the literature and is still of scientific interest (HASHEMINEJAD, RABBani, 2015; Szemela, 2015; ZaWieska et al., 2007). In many cases the sound propagates from the device to the casing structurally. It this paper acoustic propagation is assumed - the casing is vibrationally isolated from the device. 
The active control of the casing is hard due to high complexity of the system. For effective control for wide range of frequencies, a large number of actuators is required. Due to the coupling between plates, especially significant when no rigid frame is present in the casing, the system cannot be partitioned into smaller independent subsystems and thus it should be controlled as a whole (MAzur, PAWElCzYK, 2015b). However, many algorithms developed for Active Noise Control and control of single plate (LENiOWsKA, Kos, 2009; PietrzKo, 2009; MAzUr, 2013) can be directly employed for active control of the casing. For instance, commonly used feedforward FXLMS algorithms can be used (MAzur, PAwelczyK, 2015a; 2015b). Such algorithms require a reference signal taken from an error microphone, error accelerometer inside the casing, a tachometer, etc. For small casings and real devices the distance between the place where noise is generated and the casing can be too small and the noise may be not sufficiently known in advance for effective control of stochastic disturbances. Fortunately, many devices generate tonal or multitonal noise. Therefore, there are various approaches to deal with it (ElliotT, 2001; SIBIELAK et al., 2015). The noise is usually stationary or depends on the rotational speed of machine parts. Such noise can be predicted effectively and the localization of the reference sensor is not important then. However, for such cases the reference sensor is not even needed and feedback control can be successfully used.
In this paper a feedback control of the casing using the Internal Model Control (IMC) structure is investigated, which can benefit of many developments made for the feedforward control.

\section{Control algorithm}

The goal of the control algorithm is to reduce deterministic disturbances at error microphones located outside the casing. Figure 1 shows a simplified block diagram of the system. The $\mathbf{P}_{p}$ represents the vector of the primary paths, the $\mathbf{S}$ represents the matrix of the secondary paths, the $\widehat{\mathbf{S}}$ represents the matrix of the models of secondary paths, $n(i)$ is the primary noise, $\mathbf{d}(i)=\left[d_{0}(i), d_{1}(i), \ldots, d_{N_{E}-1}(i)\right]$ is the vector of disturbances at error microphones, $\widehat{\mathbf{d}}(i)=\left[\widehat{d}_{0}(i), \widehat{d}_{1}(i), \ldots, \widehat{d}_{N_{E}-1}(i)\right]$ is the vector of estimated disturbances at error microphones, $\mathbf{y}(i)$ is the undisturbed plant output vector, $\mathbf{f}$ is the reference signal selection vector, $\widehat{\mathbf{y}}(i)$ is the estimated undisturbed plant output vector and $\mathbf{e}(i)=\left[e_{0}(i), e_{1}(i), \ldots, e_{N_{E}-1}(i)\right]$ is the vector of error signals. There are $N_{E}$ error microphones, $e_{0}(i)$ to $e_{N_{E}-1}$ are signals acquired by them, and $d_{0}(i)$ to $d_{N_{E}-1}$ are disturbances at error microphones. Due to non-stationary of the plant adaptive control is used. The plant may change due to various reasons, mostly changes in the acoustic environment, but also plate temperature changes may cause huge

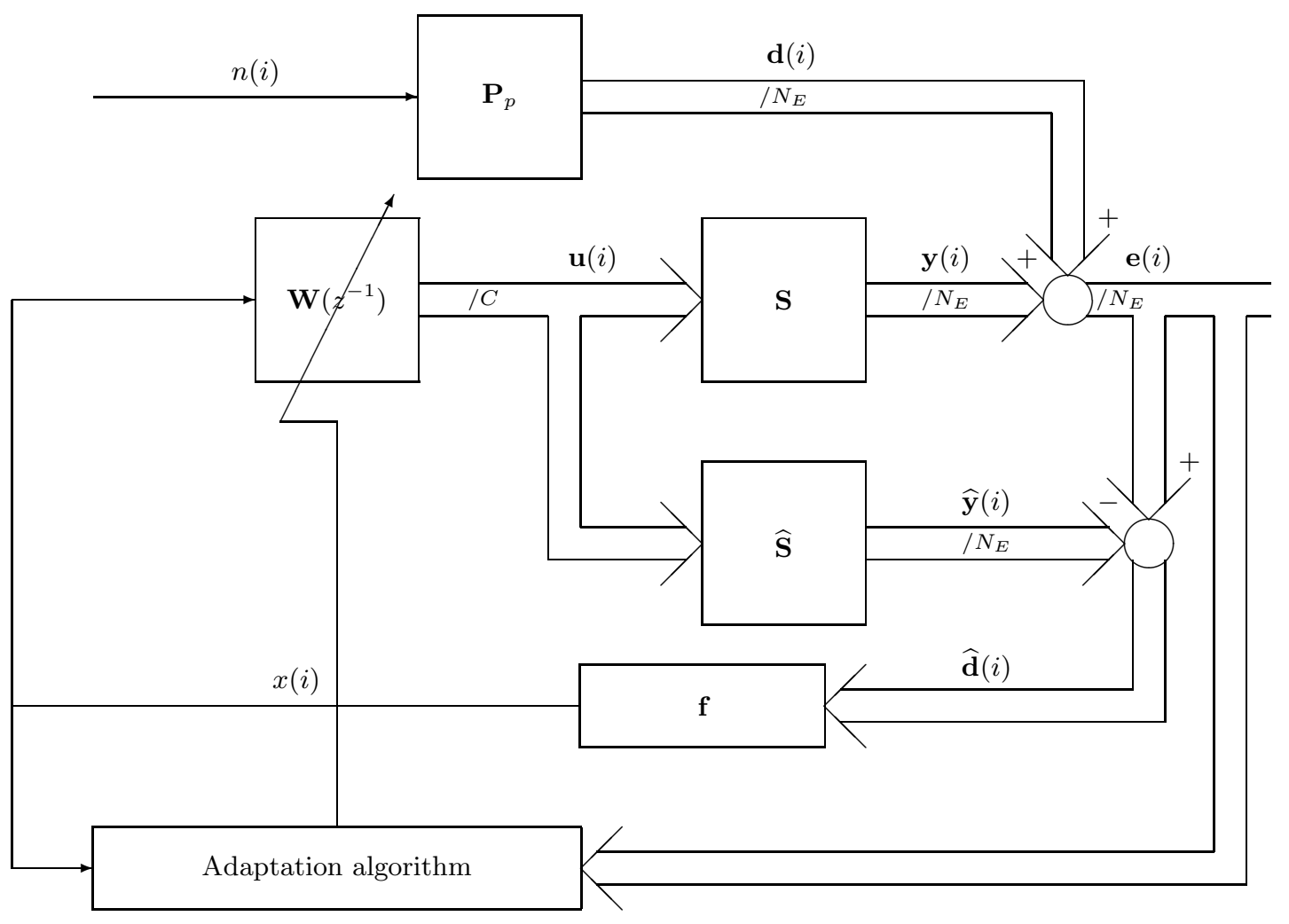

Fig. 1. IMC system block diagram for the $p$-th plate. 
changes in behavior of the plates (MAZUR, PAWELCZYK, 2011). For devices that generate a lot of heat, device casing temperature changes can be very high and even on-line adaptation of the secondary paths may be necessary. In case of the laboratory setup, adaptation of secondary paths was not necessary and the models were identified off-line.

To take advantage of parallel processing each wall is controlled by a different controller. However, all controllers share data and minimize all errors.

In the IMC structure an estimate of disturbance signals are used as reference signals. The disturbance signal for the $j$-th error microphone is estimated as:

$$
\widehat{d}_{j}(i)=e_{j}(i)-\sum_{p=0}^{P-1} \sum_{c=0}^{C_{p}-1} \widehat{\mathbf{s}}_{p, c, j}(i)^{\mathrm{T}} \mathbf{u}_{p, c}(i)
$$

where $P$ is the number of plates, $C_{p}$ is the number of actuators on the $p$-th plate, $\widehat{\mathbf{s}}_{p, c, j}(i)=$ $\left[\widehat{s}_{p, c, j, 0}(i), \widehat{s}_{p, c, j, 1}(i), \ldots, \widehat{s}_{p, c, j, N_{S}-1}(i)\right]$ is a FIR-filter estimate of the secondary path for the $c$-th output of the $p$-th plate to the $j$-th error microphone, $\mathbf{u}_{p, c}(i)=$ $\left[u_{p, c}(i), u_{p, c}(i-1), \ldots, x_{p, c}\left(i-\left(N_{S}-1\right)\right)\right]^{\mathrm{T}}$ is a vector of regressors of $c$-th output of the $p$-th plate and $N_{S}$ is the length of the FIR filters.

All estimated disturbance signals can be used as reference signals. Unfortunately, using many reference signals increases the computational complexity of the control algorithm. For feedforward control reference sensors allow for acquiring noise earlier, when there are multiple noise sources. For IMC approach applied for predictable disturbances that advantage is not important. As long as the primary noise is observed by a single error microphone, it is sufficient to use only one reference signal.

The estimated disturbance at one error microphone is used as the reference signal:

$$
x(i)=\mathbf{f}^{\mathrm{T}} \widehat{\mathbf{d}}(i),
$$

where $\mathbf{f}$ is the reference signal selection vector. In this paper $\mathbf{f}=[1,0,0,0,0]^{\mathrm{T}}$.

Each actuator is controlled by a dedicated linear FIR control filter:

$$
u_{p, c}(i+1)=\mathbf{w}_{p, c}(i)^{\mathrm{T}} \mathbf{x}_{u}(i),
$$

where $\mathbf{w}_{p, c}(i)=\left[w_{p, c, 0}(i), w_{p, c, 1}(i), \ldots, w_{p, c, N_{W}-1}(i)\right]^{\mathrm{T}}$ is a vector of parameters of the filter for the $c$-th actuator on the $p$-th plate and $N_{W}$ is the length of the control filter. $\mathbf{x}_{u}(i)=\left[x(i), x(i-1), \ldots, x\left(i-\left(N_{W}-1\right)\right)\right]^{\mathrm{T}}$ is a vector of regressors of the reference signal.

Due to high coupling between plates, for effective control and stability the plant must be controlled as a whole. The multi-error LMS (ELLIOTT et al., 1987) can be used for control. However, the number of secondary paths is very large, 105 in this case (21 outputs $\times 5$ errors), and in case of more error sensors it can be much higher. To decrease the computational demands a round-robin error switching is used instead (MichalczyK, WieczoreK, 2011; Mazur, PAWELCZYK, 2015b).

The control filters are adopted using only one error at once (ElliotT, 2001):

$$
\mathbf{w}_{p, c}(i+1)=\alpha \mathbf{w}_{p, c}(i)-\mu_{p}(i) \mathbf{r}_{p, c, v(i)}(i) e_{v(i)}(i),
$$

where $\alpha$ is the leak factor, $\mu_{p}(i)$ is the step size, $v(i)$ is the selected error, $e_{j}(i)$ is the $j$-th error signal, $j$ is set to $v(i)$ in this equation. The adaptation computational cost can be further reduced using partial update LMS algorithms (BISMOR, 2014).

The error used for adaptation is selected according to:

$$
v(i)=\left\lfloor i / N_{I}\right\rfloor \bmod N_{E},
$$

where $N_{I}$ is the number of samples, for which the error is selected.

In Eq. (4) $\mathbf{r}_{p, c, j}(i)=\left[r_{p, c, j}(i), r_{p, c, j}(i-1), \ldots\right.$, $\left.r_{p, c, j}\left(i-\left(N_{W}-1\right)\right)\right]^{\mathrm{T}}$ is a vector of regressors of the filtered-reference signal, with elements obtained as:

$$
r_{p, c, j}(i)=\widehat{\mathbf{s}}_{p, c, j}(i)^{\mathrm{T}} \mathbf{x}_{r},
$$

$\mathbf{x}_{r}=\left[x(i), x(i-1), \ldots, x\left(i-\left(N_{S}-1\right)\right)\right]^{\mathrm{T}}$ is a vector of regressors of the reference signal.

The LMS step size $\mu_{p}(i)$ is variable to adopt to different noise power levels. Many algorithms that optimize step size for faster adaptation have been developed (BISMOR et al., 2016). A popular Normalized LMS algorithm is used in this research (HAYKIN, 1996), with some modifications:

$$
\mu_{p}(i)=m(i)\left(P_{p}(i)+\zeta\right)^{-1} \mu_{n}
$$

where $\zeta$ is a small constant used to limit the step size and $m(i)$ is the mask signal equal to 0 or 1 . The mask signal is equal to 0 to disable adaptation for some time after error signal switching, so only $\mathbf{r}_{p, c, j}(i)$ vectors for single error must be calculated in each sample:

$$
m(i)=\left\{\begin{array}{l}
0\left(i \bmod N_{I}\right)<N_{S}, \\
1\left(i \bmod N_{I}\right) \geq N_{S} .
\end{array}\right.
$$

In Eq. (7) the signal power $P_{p}$ is calculated differently compared to the Normalized LMS algorithm. The power must be averaged over signals from all error sensors, not only the current error, and the Normalized LMS algorithm cannot be used directly in Eq. (4). To reduce the memory and processing power requirements, an exponential weighting is used instead of sum of squares:

$$
\begin{aligned}
P_{p}(i)= & P_{p}(i-1) \\
& +\beta\left(N_{W}\left(\sum_{l=0}^{N_{C}-1}\left(r_{p, l, v(i)}(i)\right)^{2}\right)-P_{p}(i-1)\right),
\end{aligned}
$$

where $\beta>0$ is a parameter controlling effective averaging window length. 


\section{Experimental verification}

The control system has been verified using a lightweight active casing (Fig. 2). The casing has a cuboidal shape. Its dimensions are $800 \times 630 \times 500 \mathrm{~mm}$. The casing has 5 actively controlled walls. The walls are made of $1.5 \mathrm{~mm}$ steel plates. On the bottom a passive isolation has been used. The primary noise has been generated using a loudspeaker placed inside the casing. On the casing walls, 21 actuators have been placed. Electrodynamic NXT EX-1 5 Watt exciters were used as actuators.

Table 1 shows the dimensions of all plates and the number of actuators on each plate. The locations of actuators and the number of actuators have been chosen to maximize controllability measures for all modes of individual plates up to selected frequency (WrONA, PAWELCZYK, 2016). The goal was to maximize of the minimal eigenvalue of the controllability Gramian ma- trix for all modes with frequencies up to around $300 \mathrm{~Hz}$ (first 17 modes of left/right plates, first 21 modes of front/back plates and first 25 modes of top plate). A model based on Kirchhoff thin plate model with exciters modeled as a mass connected with a spring was used. The minimal number of actuators that satisfy assumed minimal controllability index have been chosen, 5 on top wall, and 4 on other walls.

As error signals, five error microphones, Front, Back, Left, Right and Top, have been used, located $0.5 \mathrm{~m}$ away from each plate (see Fig. 2). Furthermore, four monitoring microphones, M0, M1, M2 and M3, have also been used and their positions in the laboratory room are shown in Fig. 2. Additionally a microphone inside the casing have been placed. This microphone have been for monitoring and also for the feedforward system used for comparison. The proposed IMC system have not been using this microphone.
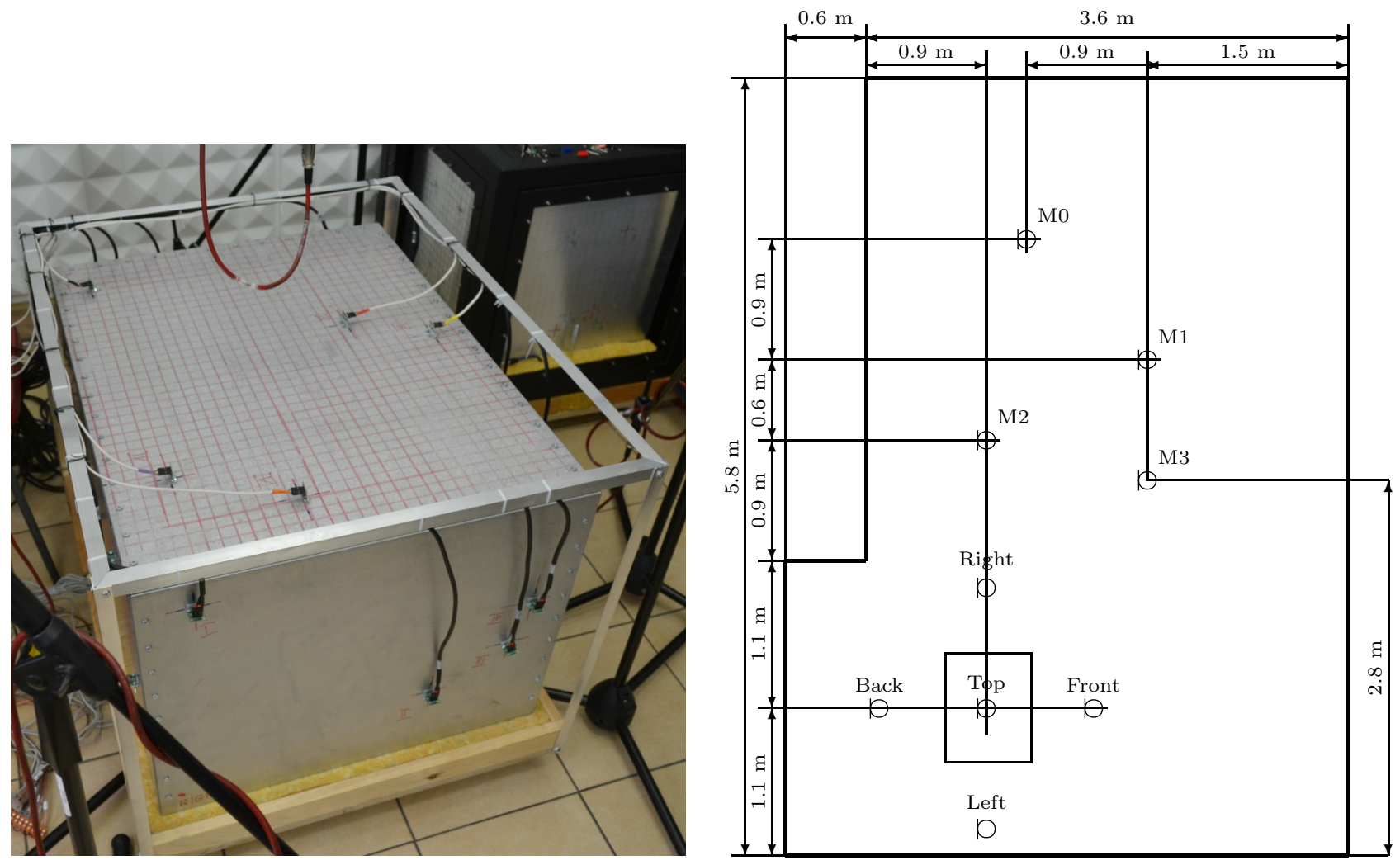

Fig. 2. The casing (left) and the position of the casing and microphones in the laboratory room (right).

Table 1. Setup details.

\begin{tabular}{|c|c|c|c|c|c|}
\hline Index & Acronym & Name & Dimensions $[\mathrm{mm}]$ & Actuators & Microphones \\
\hline 0 & $\mathrm{~F}$ & Front & $800 \times 500$ & 4 & 1 \\
\hline 1 & $\mathrm{R}$ & Right & $630 \times 500$ & 4 & 1 \\
\hline 2 & $\mathrm{~B}$ & Back & $800 \times 500$ & 4 & 1 \\
\hline 3 & L & Left & $630 \times 500$ & 4 & 1 \\
\hline 4 & T & Top & $800 \times 630$ & 5 & 1 \\
\hline
\end{tabular}


Table 2. Parameters of the control system.

\begin{tabular}{|c|c|c|c|c|c|c|c|c|c|c|}
\hline$N_{S}$ & $N_{W}$ & $N_{I}$ & $N_{E}$ & $P$ & $\mu$ & $\alpha$ & $\zeta$ & $\beta$ & $C_{0}$ to $C_{3}$ & $C_{4}$ \\
\hline 128 & 80 & 256 & 5 & 5 & 0.001 & 0.9999999 & $10^{-6}$ & $10^{-4}$ & 4 & 5 \\
\hline
\end{tabular}

Table 2 shows parameters of the control system. The control system have used 5 DS1104 boards, one board per plate. The boards communicate with each other through a Peripheral Component Interconnect (PCI) bus. The algorithm has operated at $2 \mathrm{kHz}$ sampling frequency. Sampling on all boards have been synchronized using an external trigger generated by one of the boards.
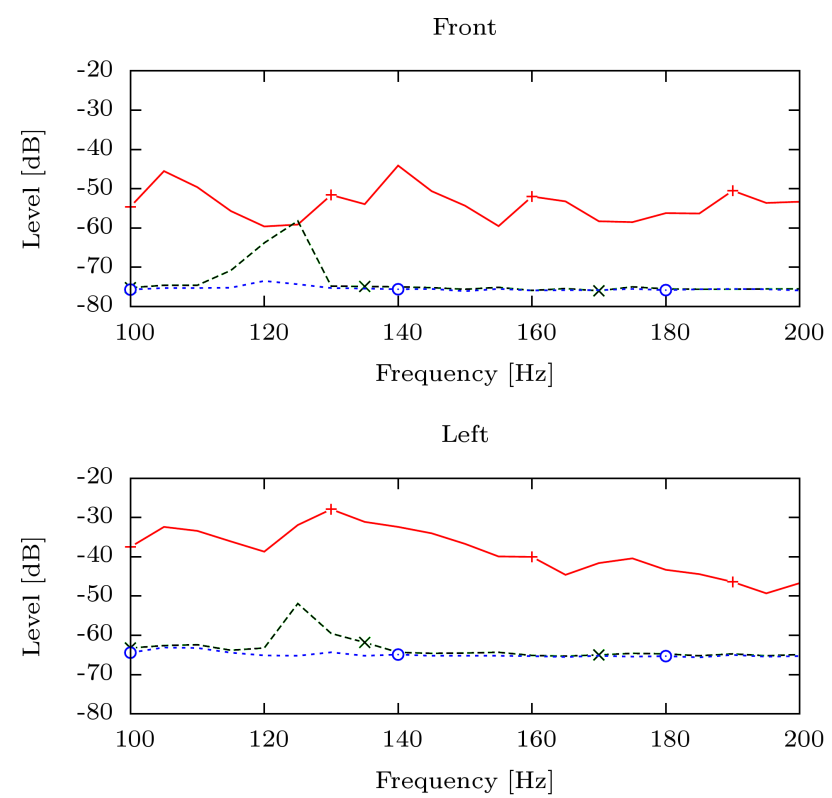

M0

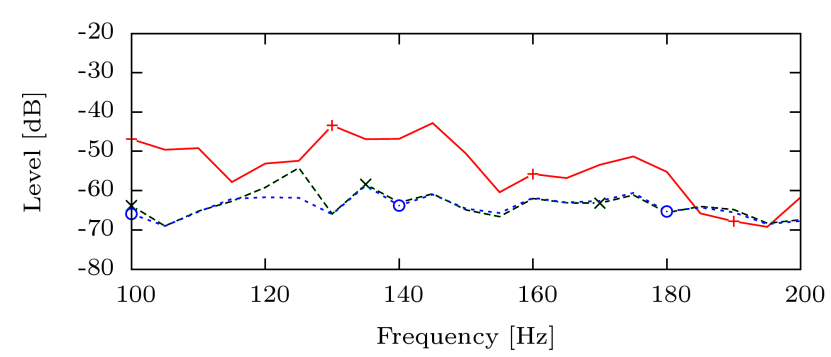

M2

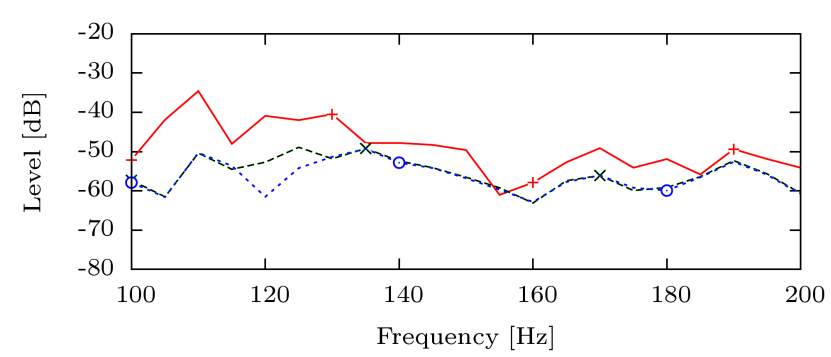

without ANC $\longrightarrow$
Figure 3 shows the error and monitoring microphones signal levels with and without ANC for tonal disturbance excitations of frequencies from $100 \mathrm{~Hz}$ to $200 \mathrm{~Hz}$. Except for some frequencies, with active control the noise was reduced to the acoustic noise floor. For most frequencies the signal level reduction at error microphones exceeds $20 \mathrm{~dB}$, and in some cases exceeds $30 \mathrm{~dB}$. The average signal level reduction at error
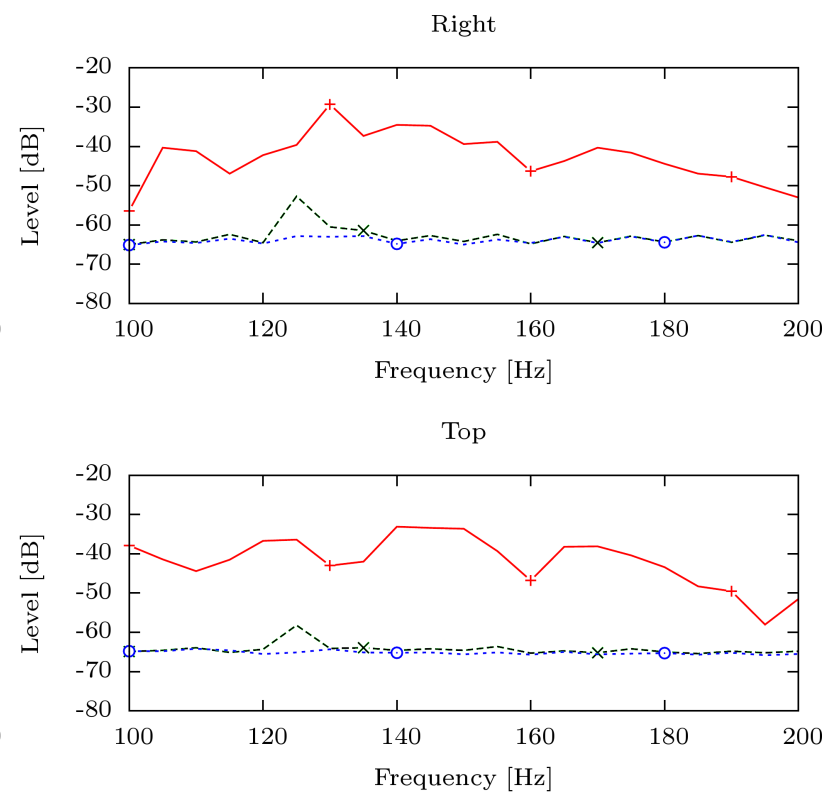

M1

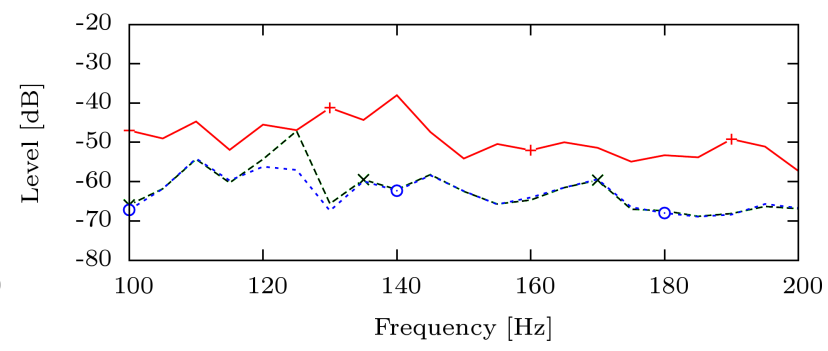

M3

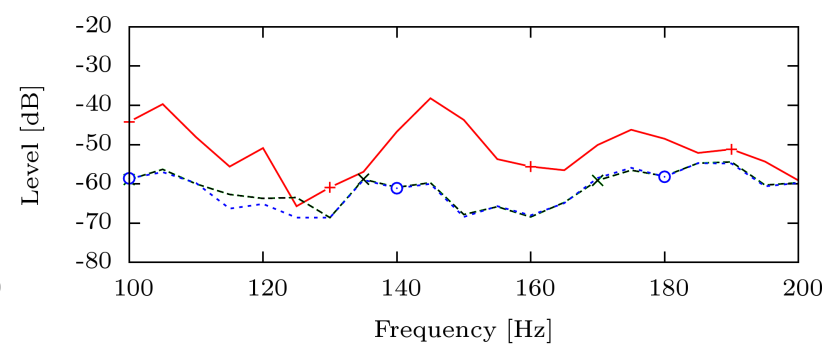

feedforward $\cdots \cdot \odot \cdots$

Fig. 3. Microphone signal levels with and without ANC for pure tonal disturbance excitations of frequencies from $100 \mathrm{~Hz}$ to $200 \mathrm{~Hz}$, with $5 \mathrm{~Hz}$ step (linearly interpolated for clarity), in subfigure title the microphone signal origin is given. 
for microphones is $23.8 \mathrm{~dB}$ for IMC, and $25.5 \mathrm{~dB}$ for feed-forward control. At monitoring microphones the average signal level reduction is $10.4 \mathrm{~dB}$ for IMC and $11.3 \mathrm{~dB}$ for feed-forward control.
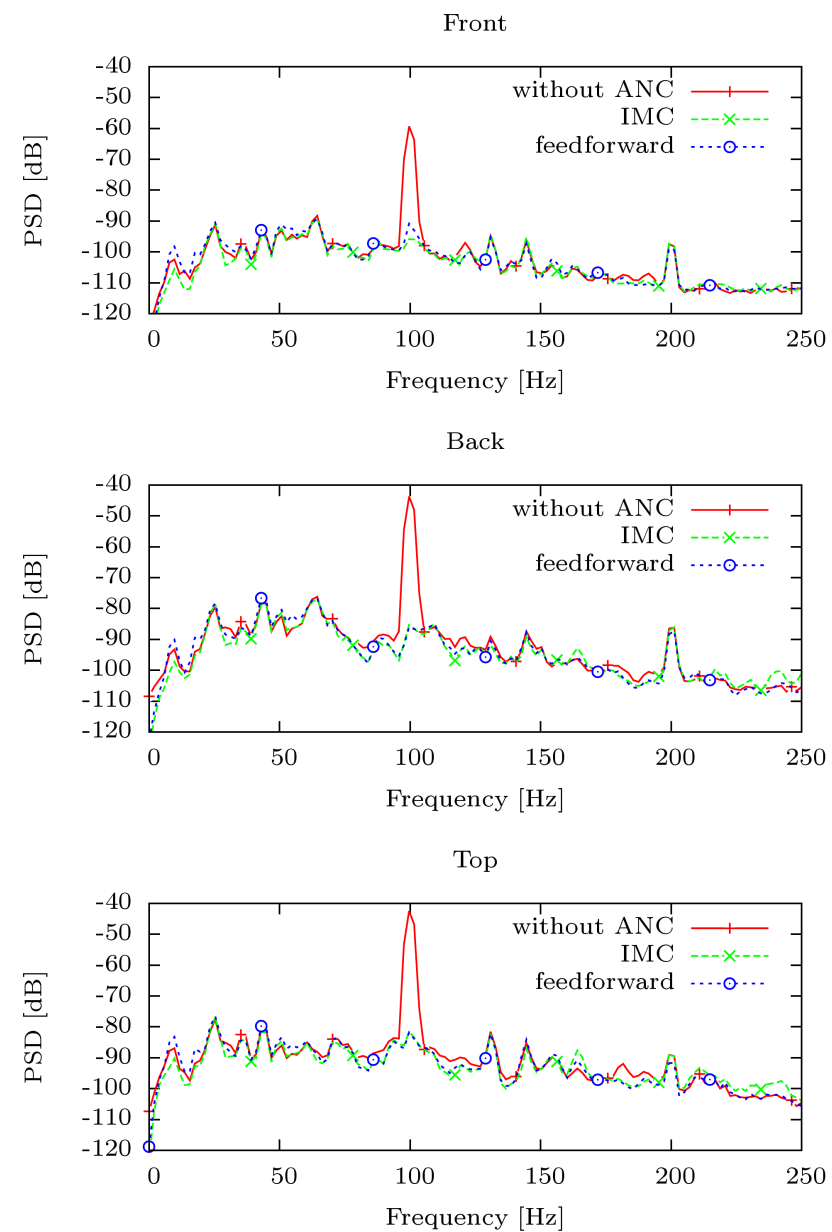

M0

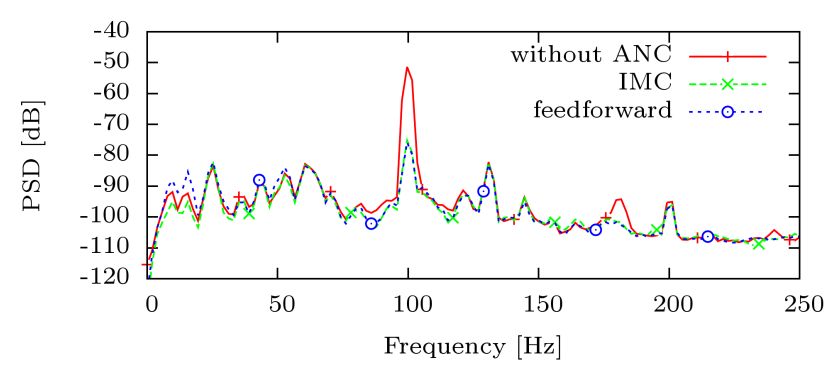

M2

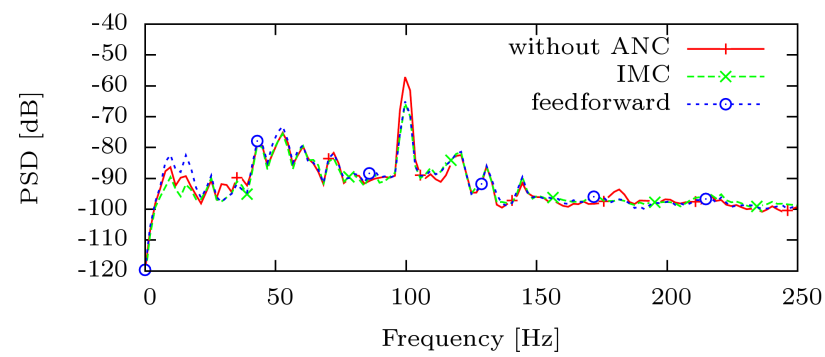

Figure 4 shows the power spectral density estimates for monitor and error microphone signals, using a $100 \mathrm{~Hz}$ tonal noise excitation, with and without ANC. At error microphones the average signal level
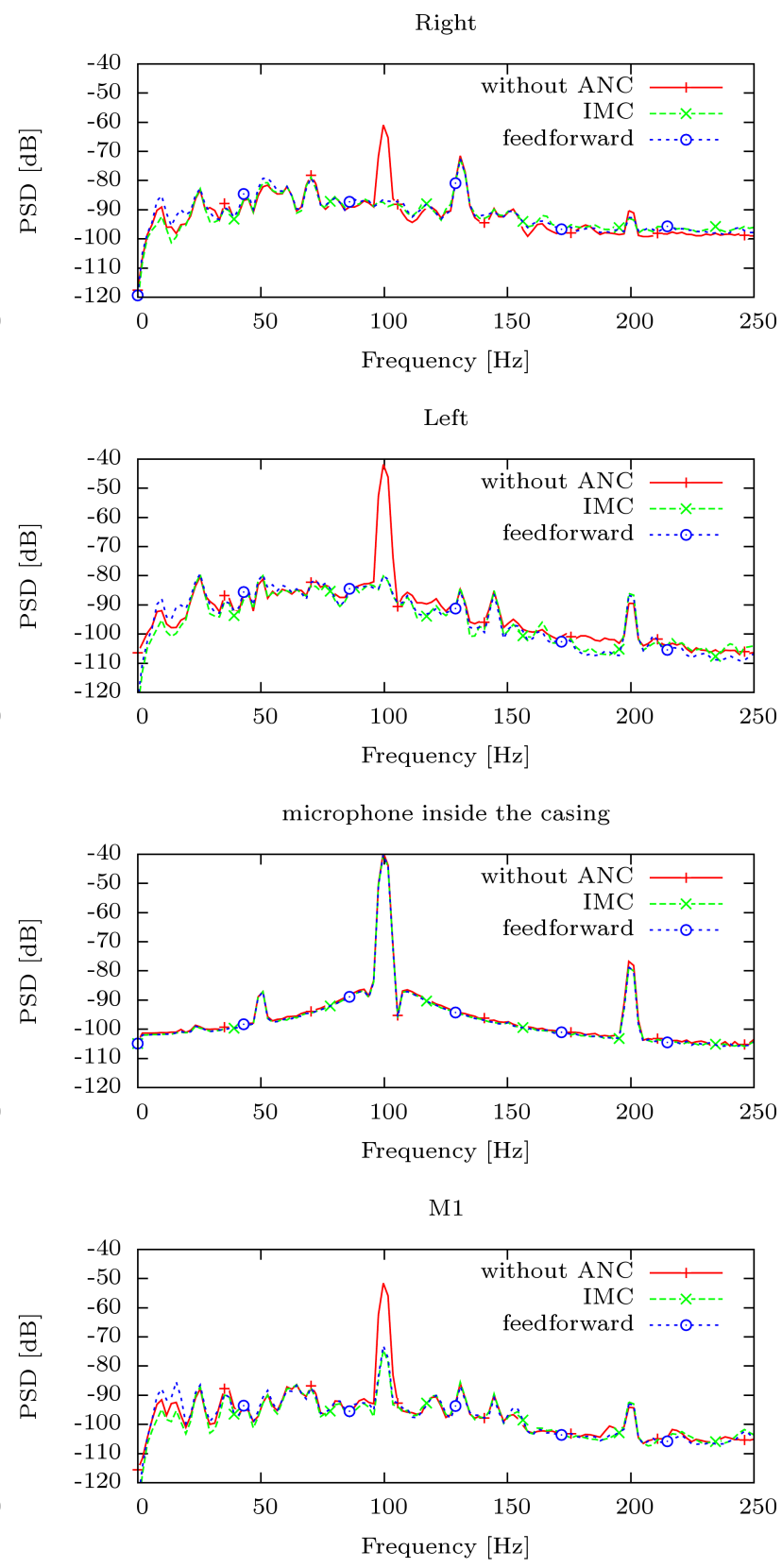

M3

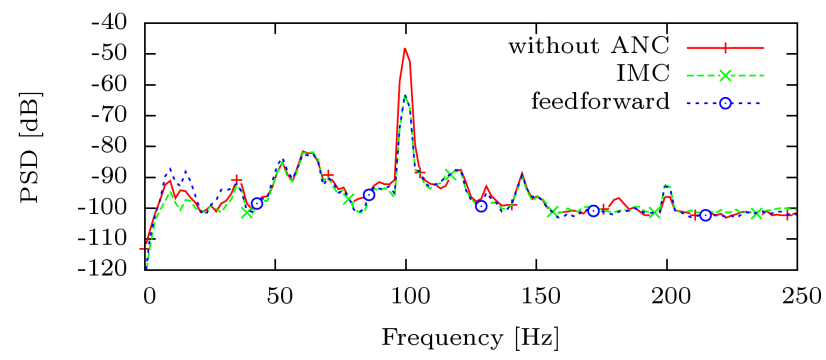

Fig. 4. Power spectral densities of microphone signals for a $100 \mathrm{~Hz}$ tonal noise excitation with and without ANC, in subfigure title the microphone signal origin is given. 
reduction is $23.8 \mathrm{~dB}$ for IMC, and $23.2 \mathrm{~dB}$ for feedforward control. At monitoring microphones the average signal level reduction is $15.1 \mathrm{~dB}$ for IMC control, and $14.5 \mathrm{~dB}$ for feed-forward control. The effects of a small plant nonlinearity are also visible, the second harmonics at $200 \mathrm{~Hz}$ is visible on some signals. In further research the measurement uncertainty will be analysed (Wiora et al., 2016).

\section{Conclusions}

Internal Model Control has been applied to the light-weight noise reducing casing. It allows to employ adaptive LMS-based algorithms originally developed for feedforward systems. Its benefit is that it does not require a reference sensor, which might be difficult to install for many applications. To guarantee stable operation and make the implementation feasible for the complicated multichannel system with a number of interactions, appropriate solutions have been proposed.

For simple tonal or multitonal disturbances the IMC with the proposed algorithm has turned out to be very effective, at error microphones the average signal level reduction exceeds $20 \mathrm{~dB}$ at error microphones and $10 \mathrm{~dB}$ at monitoring microphones. The IMC system is able to reduce noise at error microphones to the acoustic noise floor level. However, at monitoring microphones the noise is still clearly visible. The results are similar to those obtained with the feedforward approach. Such deterministic noise is very common and for such applications the IMC system is an attractive approach.

\section{Acknowledgment}

The research reported in this paper has been supported by the National Science Centre, Poland, decision no. DEC-2012/07/B/ST7/01408.

The authors would like to thank two anonymous reviewers for their valuable comments, which helped to improve the paper.

\section{References}

1. Bismor D. (2014), Partial Update LMS Algorithms in Active Noise Control, [in:] Proceedings of the Forum Acusticum 2014, Kraków.

2. Bismor D., Czyz K., Ogonowski Z. (2016), Review and Comparison of Variable Step-Size LMS Algorithms, International Journal of Acoustics and Vibration, 21, 1.

3. Elliott S.E., Stothers I.M., Nelson P.A. (1987), A Multiple Error LMS Algorithm and Its Application to the Active Control of Sound and Vibration, IEEE Transations on Acoustics, Speech and Signal Processing, ASSP-35, 10, 1423-1434.
4. ElLiott S.J. (2001), Signal Processing for Active Control, Academic Press, London.

5. Fuller C., Mcloughlin M., Hildebrand S. (1994) (Apr. 28), Active acoustic transmission loss box, WO Patent App. PCT/US1992/008,401.

6. Górski P., Kozupa M. (2012), Variable Sound Insulation Structure with MFC Elements, Archives of Acoustics, 37, 1, 115-120.

7. Hasheminejad S.M., Rabbani V. (2015), Active Transient Sound Radiation Control from a Smart Piezocomposite Hollow Cylinder, Archives of Acoustics, 40, 3, 359-381.

8. Haykin S.S. (1996), Adaptive Filter Theory, PrenticeHall information and system sciences series, Prentice Hall.

9. Leniowska L., Kos P. (2009), Self-tuning control with regularized $R L S$ algorithm for vibration cancellation of a circular plate, Archives of Acoustics, 34, 4, 613-624.

10. Leniowska L., Mazan D. (2015), MFC Sensors and Actuators in Active Vibration Control of the Circular Plate, Archives of Acoustics, 40, 2, 257-265.

11. MAzur K. (2013), Active control of sound with a vibrating plate, Ph.D. thesis, Silesian University of Technology, Gliwice, Poland.

12. Mazur K., Pawelczyk M. (2011), Active noisevibration control using the filtered-reference LMS algorithm with compensation of vibrating plate temperature variation, Archives of Acoustics, 36, 1, 65-76.

13. Mazur K., Pawelczyk M. (2013a), Active Noise Control with a single nonlinear control filter for a vibrating plate with multiple actuators., Archives of Acoustics, 38, 4.

14. Mazur K., Pawelczyk M. (2013b), Hammerstein nonlinear active noise control with the Filtered-Error LMS algorithm, Archives of Acoustics, 38, 2, 197-203.

15. Mazur K., Pawelczyk M. (2015a), Active control of noise emitted from a device casing, [in:] Proceedings of the 22nd International Congress of Sound and Vibration, Florence, Italy.

16. Mazur K., Pawelczyk M. (2015b), Multiple-error adaptive control of an active noise-reducing casing, [in:] Progress of Acoustics, pp. 701-712, Polish Acoustical Society, Wrocław.

17. Michalczyk M., Wieczorek M. (2011), Parameterization of adaptive control algorithms for multi-channel active noise control system, [in:] 58th Open Seminar on Acoustics joint with 2nd Polish-German Structured Conference on Acoustics, Jurata, Poland 1316.09.2011, pp. 73-78, vol. 2.

18. Pietrzko S.J. (2009), Contributions to Noise and Vibration Control Technology, AGH - University of Science and Technology Press, Kraków.

19. Shehap A.M., Shawky H.A., El-Basheer T.M. (2016), Study and Assessment of Low Frequency Noise in Occupational Settings, Archives of Acoustics, 41, 1, 151-160. 
20. Sibielak M., Raczka W., Konieczny J., Kowal J. (2015), Optimal control based on a modified quadratic performance index for systems disturbed by sinusoidal signals, Mechanical Systems and Signal Processing, 64, 498-519.

21. Szemela K. (2015), Sound Radiation from a Surface Source Located at the Bottom of the Wedge Region, Archives of Acoustics, 40, 2, 223-234.

22. Wiora J., Kozyra A., Wiora A. (2016), A weighted method for reducing measurement uncertainty below that which results from maximum permissible error, Measurement Science and Technology, 27.

23. Wrona S., PAwelczyk M. (2014), Active reduction of device multi-tonal noise by controlling vibration of multiple walls of the device casing, [in:] Proceedings of the 19th International Conference on Methods and Models in Automation and Robotics (MMAR), Miedzyzdroje, Poland, pp. 687-692.

24. Wrona S., Pawelczyk M. (2016), Optimal placement of actuators for Active Structural Acoustic Control of a light-weight device casing, [in:] Proceedings of the 23nd International Congress of Sound and Vibration, Athens, Greece.

25. Wrona S., Pawelczyk M., Wyrwal J. (2014), Optimal placement of elastically mounted vibration actuators on a plate, for active noise-vibration control, [in:] Proceedings of 18th National Conference on Automatic Control, 8-10 September, Wroclaw, Poland.

26. Zawieska W.M., Rdzanek W.P., Rdzanek W.J., ENGEL Z. (2007), Low frequency estimation for the sound radiation efficiency of some simply supported flat plates, Acta Acustica United with Acustica, 93, 3, 353363. 